\title{
Restart of Anticoagulant Therapy and Risk of Thrombosis, Rebleeding, and Death after Factor Xa Inhibitor Reversal in Major Bleeding Patients
}

\author{
Truman J. Milling Jr. ${ }^{1}$ Ben King ${ }^{2}$ Patrick Yue ${ }^{3}$ Saskia Middeldorp ${ }^{4,5}$ Jan Beyer-Westendorf ${ }^{6}$ \\ John W. Eikelboom ${ }^{7}$ Mark Crowther ${ }^{7}$ Lizhen $\mathrm{Xu}^{8} \quad$ Peter Verhamme $^{9 \odot}$ Deborah M. Siegal ${ }^{10}$ \\ Stuart J. Connolly ${ }^{8}$ and on behalf of the ANNEXA-4 Investigators
}

${ }^{1}$ Department of Neurology, Seton Dell Medical School Stroke Institute, Austin, Texas, United States

2 Department of Health Systems and Population Health Sciences, University of Houston College of Medicine, Houston, Texas, United States

3 Portola Pharmaceuticals, Inc., now Alexion Pharmaceuticals, Inc., South San Francisco, California, United States

${ }^{4}$ Department of Internal Medicine, Radboud University Medical Center, Nijmegen, The Netherlands

${ }^{5}$ Radboud Institute for Health Sciences, Radboud University Medical Center, Nijmegen, The Netherlands

${ }^{6}$ Division of Hematology and Hemostasis, Department of Medicine I, University Hospital Dresden, Dresden, Germany

7 Department of Medicine, McMaster University, Hamilton, Ontario, Canada

8 Population Health Research Institute, McMaster University, Hamilton, Ontario, Canada

${ }^{9}$ Center for Molecular and Vascular Biology, University of Leuven, Leuven, Belgium

10 Department of Medicine, University of Ottawa, Ottawa, Ontario, Canada

Thromb Haemost 2021;121:1097-1106.
Address for correspondence Truman J. Milling Jr., MD, Seton Dell Medical School Stroke Institute, 1601 Trinity Street, 10th Floor, Austin, TX 78712, United States (e-mail: Tmilling@ascension.org).

\section{Abstract \\ Keywords \\ - pulmonary embolism \\ - stroke \\ - prevention \\ - thrombosis \\ - venous thromboembolism}

Background Lack of data on balancing bleeding and thrombosis risk causes uncertainty about restarting anticoagulants after major bleeding. Anticoagulant reversal trials offer prospectively gathered data after major bleeding with well-documented safety events and restarting behavior.

Objectives To examine the relationship of restarting anticoagulation with thrombosis, rebleeding, and death.

Methods This is a posthoc analysis of a prospective factor Xa inhibitor reversal study at 63 centers in North America and Europe. We compared outcomes of restarted patients with those not restarted using landmark and time-dependent Cox proportional hazards models. Outcomes included thrombotic and bleeding events and death and a composite of all three. received

January 19, 2021

accepted after revision

February 23, 2021

published online

February 25, 2021
DOI https://doi.org/ 10.1055/a-1400-6159. ISSN 0340-6245. (c) 2021. The Author(s).

This is an open access article published by Thieme under the terms of the Creative Commons Attribution-NonDerivative-NonCommercial-License, permitting copying and reproduction so long as the original work is given appropriate credit. Contents may not be used for commercial purposes, or adapted, remixed, transformed or built upon. (https://creativecommons.org/ licenses/by-nc-nd/4.0/) Georg Thieme Verlag KG, Rüdigerstraße 14, 70469 Stuttgart, Germany 
Results Of 352 patients enrolled, oral anticoagulation was restarted in $100(28 \%)$ during 30-day follow-up. Thirty-four (9.7\%) had thrombotic events, 15 (4.3\%) had bleeding events (after day 3 ), and 49 (14\%) died. In the landmark analysis comparing patients restarted within 14 days to those not, restarting was associated with decreased thrombotic events (hazard ratio $[\mathrm{HR}]=0.112 ; 95 \%$ confidence interval $[\mathrm{Cl}]$ : $0.001-0.944 ; p=0.043$ ) and increased rebleeding ( $\mathrm{HR}=8.39 ; 95 \% \mathrm{Cl}: 1.13-62.29 ; p=0.037)$. The time-dependent Cox model showed evidence for a reduction in a composite (thrombotic events, bleeding, and death) attempting to capture net benefit ( $\mathrm{HR}=0.384 ; 95 \% \mathrm{Cl}: 0.161-0.915 ; p=0.031)$. Conclusion This analysis provides modest evidence that restarting anticoagulation in factor Xa inhibitor-associated major bleeding patients is correlated with reduced risk of thrombotic events and increased risk of rebleeding. There is low-level evidence of net benefit for restarting. A randomized trial of restarting would be appropriate.

\section{Introduction}

Postacute care of anticoagulant-associated major hemorrhage is a challenging clinical scenario as there is significant uncertainty about whether and when to restart anticoagulants. Recently, researchers have focused on early anticoagulation restart after anticoagulant-associated major hemorrhage as a strategy to reduce thrombotic events. ${ }^{1,2}$ Several retrospective analyses, registry studies, and metaanalyses support restarting, mostly in atrial fibrillation populations, to reduce thrombotic events, though with some increase in rebleeding. ${ }^{3-8}$ The ANNEXA-4 study of patients with factor Xa (FXa) inhibitor-associated severe and potentially life-threatening bleeding receiving andexanet alfa was performed at 63 medical centers in North America and Europe, leading to regulatory approval. ${ }^{9}$ ANNEXA-4 data provide an opportunity to study prospectively gathered data on 352 patients with FXa inhibitorassociated major bleeding, many of whom were restarted on oral anticoagulation at the discretion of their treating physicians.

We previously reported in the ANNEXA-4 trial that no thrombotic events occurred after restart of therapeutic oral anticoagulation, ${ }^{9}$ but did not examine the association of restarting with bleeding or death or with any composites that attempt to detect a net clinical benefit. The ANNEXA-4 dataset offers granular details on anticoagulant restarting behavior, thrombotic and bleeding events, and death through 30 days with very few patient dropouts. This allows more thorough analysis than retrospective hospital datasets which often lose track of patients at discharge, and thus fail to capture subsequent thrombotic and bleeding events. The present study is a posthoc analysis evaluating the association between the occurrence of thrombotic events, rebleeding, and death with the restarting of oral anticoagulation in patients with FXa inhibitor-associated major bleeding.

\section{Methods}

The ANNEXA-4 study and patient population have been previously described. ${ }^{9}$ Briefly, patients presented with FXa inhibitor-associated major bleeding, of which, 227 (64\%) had intracranial hemorrhage (ICH; 128 spontaneous, 99 traumatic), 90 (26\%) had gastrointestinal (GI) bleeding, and 35 $(10 \%)$ had other major bleeding ( - Table $\mathbf{1}$ ). Baseline characteristics, such as demographics, bleeding site, anti-FXa activity, indication for anticoagulation, $\mathrm{CHA}_{2} \mathrm{DS}_{2}$-VASc score, history of thromboembolism and bleeding, and time from last dose to bleeding onset, were assessed.

The follow-up started on the day of hemorrhage and ended 30 days later. For this analysis, restart of anticoagulation was defined as administration of therapeutic dose oral anticoagulants. The decision to restart anticoagulation was made by the treating physician based on clinical judgment for each patient and could occur during the hospital stay or at any time to 30-day end of study. We did not examine use of other forms of anticoagulation, such as prophylactic dose low-molecular-weight heparin administered for venous thromboembolism (VTE) prevention during hospitalization. Four outcomes were assessed: thrombotic events, rebleeding, a composite of thrombotic events or rebleeding, and a composite of thrombotic events, rebleeding, or death, which was designed to represent a net clinical benefit. Thrombotic events were primary safety outcomes in ANNEXA-4, i.e., VTE, ischemic stroke, other systemic embolism, and myocardial infarction. Symptomatic thrombotic events were reported by site investigators and adjudicated by an independent academic committee. Number and types of thrombotic events have been previously published. ${ }^{9}$

Rebleeding events were defined as bleeding at any site severe enough to be categorized by the investigator as a serious adverse event (SAE; e.g., death, a life-threatening, inpatient hospitalization, prolongation of existing hospitalization). Bleeding events were evaluated posthoc for whether they represented true bleeding complications by two independent reviewers (T.J.M. and J.W.E.), and any disagreements were adjudicated by a third reviewer (M.C.). This was done by reviewing all SAEs related to bleeding and applying clinical judgment as to whether they represented a bleeding event. Although reviewers could not remain entirely agnostic to whether the patient had been restarted on anticoagulation 
as it was sometimes mentioned in the SAE report, they were asked only to determine whether a bleeding event had occurred, not to establish relatedness.

Baseline characteristics were summarized by patient groups defined by whether anticoagulation was restarted or not. Categorical variables were reported as counts (percentages) and compared between the two groups by Pearson's chi-squared test. Continuous variables were summarized as mean (standard deviation) and/or median (interquartile range [IQR]) and compared between groups using the Wilcoxon rank-sum test. Statistical significance was determined at the two-sided $\alpha=0.05$. Variables identified as significantly different between the two groups ( - Table 1) were included in all multivariate Cox models.

As anticoagulation restart could occur any time during follow-up, the exposure variable is time-dependent. As a first step, we conducted overall (naïve) tests of the relationship between restart and various outcomes irrespective of the

Table 1 Baseline characteristics for anticoagulation restart and non-restart (red for those controlled for multivariate analysis)

\begin{tabular}{|c|c|c|c|c|}
\hline Variable & Total $(N=352)$ & OAC non-restart $(n=252)$ & OAC restart $(n=100)$ & $p$-Value \\
\hline \multicolumn{5}{|l|}{ Age, y } \\
\hline Median (IQR) & $79.0(72.0-84.0)$ & $80.0(73.0-86.0)$ & $76.5(71.0-82.0)$ & 0.005 \\
\hline Mean (SD) & $77.36(10.80)$ & $78.38(10.41)$ & $74.79(11.36)$ & \\
\hline Race, No. (\%) & & & & 0.171 \\
\hline White & $307(87.2)$ & $223(88.5)$ & $84(84.0)$ & \\
\hline Black/African-American & $29(8.2)$ & $17(6.7)$ & $12(12.0)$ & \\
\hline Other & $7(2.0)$ & $4(1.6)$ & $3(3.0)$ & \\
\hline \multicolumn{5}{|l|}{ Ethnicity, No. (\%) } \\
\hline Non-Hispanic & $327(92.9)$ & $231(91.7)$ & $96(96.0)$ & 0.125 \\
\hline \multicolumn{5}{|l|}{ Sex, No. (\%) } \\
\hline Male & $187(53.1)$ & $130(51.6)$ & $57(57.0)$ & 0.359 \\
\hline \multicolumn{5}{|l|}{ Primary bleed site, No. (\%) } \\
\hline $\mathrm{ICrH}$ & $227(64.5)$ & $186(73.8)$ & $41(41.0)$ & $<0.001$ \\
\hline $\mathrm{Gl}$ & $90(25.6)$ & $50(19.8)$ & $40(40.0)$ & $<0.001$ \\
\hline Other & $35(9.9)$ & $16(6.3)$ & $19(19.0)$ & $<0.001$ \\
\hline \multicolumn{5}{|l|}{ Indication for FXal, No. (\%) } \\
\hline AF & $280(79.5)$ & $204(81.0)$ & $76(76.0)$ & 0.299 \\
\hline VTE & $71(20.2)$ & $47(18.7)$ & $24(24.0)$ & 0.259 \\
\hline Other & $26(7.4)$ & $20(7.9)$ & $6(6.0)$ & 0.531 \\
\hline $\begin{array}{l}\text { Positive history of bleeding in the } \\
\text { past } 6 \text { months, No. (\%) }\end{array}$ & $25(7.1)$ & $17(6.7)$ & $8(8.0)$ & 0.701 \\
\hline \multicolumn{5}{|c|}{ History of any thrombotic events in the past 6 months, No. (\%) } \\
\hline $\mathrm{Ml}$ & $8(2.3)$ & $5(2.0)$ & $3(3.0)$ & 0.693 \\
\hline Stroke & $305(86.6)$ & $217(86.1)$ & $88(88.0)$ & 0.762 \\
\hline TIA & $332(94.3)$ & $235(93.3)$ & $97(97.0)$ & 0.168 \\
\hline Angina & $24(6.8)$ & $15(6.0)$ & $9(9.0)$ & 0.306 \\
\hline VTE & $38(10.8)$ & $31(12.3)$ & $7(7.0)$ & 0.156 \\
\hline Antiplatelet medications, No. (\%) & $92(26.1)$ & $64(25.4)$ & $28(28.0)$ & 0.616 \\
\hline Bleeding is a result of trauma, No. (\%) & $113(32.1)$ & $82(32.5)$ & $31(31.0)$ & 0.780 \\
\hline $\begin{array}{l}\text { Time from the last FXal dose to the } \\
\text { onset of bleeding, } n^{\text {a }}\end{array}$ & 163 & 110 & 53 & \\
\hline$>8 \mathrm{~h}$, No. (\%) & $43(26.4)$ & $30(27.3)$ & $13(24.5)$ & 0.710 \\
\hline CHA2DS2-VASc score, $n$ & 352 & 252 & 100 & \\
\hline Median (IQR) & $4.0(3.0-6.0)$ & $4.0(3.0-6.0)$ & $4.0(3.0-5.0)$ & 0.198 \\
\hline
\end{tabular}

Abbreviations: AF, atrial fibrillation; FXal, factor Xa inhibitor; Gl, gastrointestinal; ICrH, intracranial hemorrhage; IQR, interquartile range; MI, myocardial infarction; OAC, oral anticoagulation; TIA, transient ischemic attack; VTE, venous thromboembolism.

a Percentages are based on number of patients with available data $(n)$ in the corresponding variable as the denominator (the denominator is at the top of the column). 
time of restart. In this univariate analysis, we used a twosided, log-rank test to compare the two treatment groups for the survival distributions of the events. Cox proportional hazards regression with the restart indicator as the only predictor was performed to estimate the hazard ratio (HR). The $95 \%$ confidence interval $(\mathrm{CI})$ was constructed by inverting the partial-likelihood score test under the Cox model. ${ }^{10}$ For the test of thrombotic events, in which a quasi-complete separation feature presented (i.e., no thrombotic events after restart), Peto's method ${ }^{11}$ was used to estimate HR and CI based on the log-rank test result. Multivariate Cox proportional hazards regression analysis was also performed to adjust for possible confounders. In the Cox regression models, parameter estimations were obtained using a traditional maximum partial likelihood method. When quasi-complete separation occurred for any analysis where no events occurred in one exposure group (such as after restart), the Firth penalized partial likelihood method ${ }^{12,13}$ for rare events was used to correct the bias. Statistical analyses were performed using SAS software, version 9.4, and R Statistical Software. ${ }^{14}$

To account for the time-dependent nature of the restart treatment and to eliminate the guarantee-time bias that it might bring, ${ }^{15}$ we then applied landmark analysis and timedependent covariate Cox regression methods to examine the association between anticoagulation restart timing and outcomes.

\section{Landmark Analysis}

Landmark analysis is a commonly used approach to avoid the guarantee-time bias induced by the time-dependent nature of the treatment. ${ }^{16}$ This approach establishes a landmark time point during the follow-up period and examines patients who are event free (no bleeding after day 3 and no thrombotic event or death) prior to the landmark for a relationship between exposures occurring prior to landmark (such as restarting) and outcomes occurring afterward (such as thrombotic or bleeding events or death). ${ }^{17}$ In our analysis, landmark time points were set a priori at day 3, 5, and 14 within the 30-day follow-up period. The earliest landmark was chosen based on prior work suggesting most rebleeding events occur within 3 days of index bleeding, ${ }^{1}$ but few patients were restarted by day 3 . We anticipated in our statistical analysis plan that this would limit our ability to detect differences in outcomes. The day 5 landmark was an approximation slightly later to allow for more restarted patients. The day 14 landmark was chosen because it roughly bisected the follow-up period. We suspected this would give it the most power to detect differences. Later landmarks would reduce power as they reduce the period of study between the landmark and the 30-day end of surveillance and thus the number of outcome events. Later landmarks also exclude more patients, i.e., those with events prior to the landmark, reducing power.

For each landmark analysis, patients were divided into restarted and non-restarted groups based on whether they had restarted therapeutic oral anticoagulation prior to the landmark time. Kaplan-Meier survival curves stratified by whether oral anticoagulation was restarted were generated.
A two-sided, log-rank test was applied to compare the survival distribution of the events. Similar to the overall (naïve) analysis, we applied both univariate (the restart indicator as the only predictor) and multivariate Cox proportional hazards regression to obtain the unadjusted and adjusted estimations of the HRs, $p$-values, and 95\% CIs.

\section{Time-Dependent Cox Regression}

Time-dependent Cox hazard models restructured the exposure data to strictly account for time spent restarted and not restarted on anticoagulation. In this model, exposure to anticoagulation restart was regarded as a time-dependent variable. It was initially assigned to be zero and irreversibly attained the value of one as soon as the patient received therapeutic oral anticoagulation treatment. We applied the multivariate Cox proportional hazards model and adjusted for the identified potential confounders to investigate the relative risk of the various events between treatment groups.

We ignored the bleeding events in the first 3 days in both models because nearly all were complications of the index bleeding events, and they confounded the model (details below).

\section{Subgroups}

We performed the same analyses on the $\mathrm{ICH}$ subgroup $(n=227)$.

\section{Results}

Restarting: Treating physicians restarted oral anticoagulation in 100 of 352 (28\%) patients in the 30-day follow-up period. Of the 100 patients who were restarted, the median restart time was 10 days (IQR: 5-16 days; - Supplementary Table S1 [available in the online version]) after the index bleeding event. Twelve patients restarted within 3 days; 30 within 5 days; and 67 within 14 days. Patients were primarily restarted on direct oral anticoagulants: $48 \%$ apixaban, $29 \%$ rivaroxaban, $4 \%$ dabigatran, and $2 \%$ edoxaban. The remaining $17 \%$ were restarted on vitamin $\mathrm{K}$ antagonists (see -Supplementary Table S2 [available in the online version] for doses/percentages, - Supplementary Table $\mathbf{5 3}$ [available in the online version] for a full list and day of restart, and - Supplementary Table S4 [available in the online version] for baseline break down of outcomes by restarted or not groups).

Patients who restarted were younger (median: 76.5 vs. 80.0 years; $p=0.005)$ and less likely to have ICH $(41 / 100 ; 41 \%$ of restarted had ICH vs. $186 / 252 ; 73.8 \%$ of not restarted had ICH; $p<0.001)$. No other baseline characteristics were significantly associated with the decision to restart using a $p$ value cut-off of 0.10 . Thus, we included both age and site of index bleeding as covariates in all multivariate Cox proportional hazards models.

Thrombotic events: Of 352 patients, 34 (9.7\%) developed at least one thrombotic event in the 30-day surveillance period. Of these 34 patients, the median time to first thrombotic event was 10 days (IQR: 2-18 days; -Supplementary Table S1 [available in the online version]). Ischemic strokes 


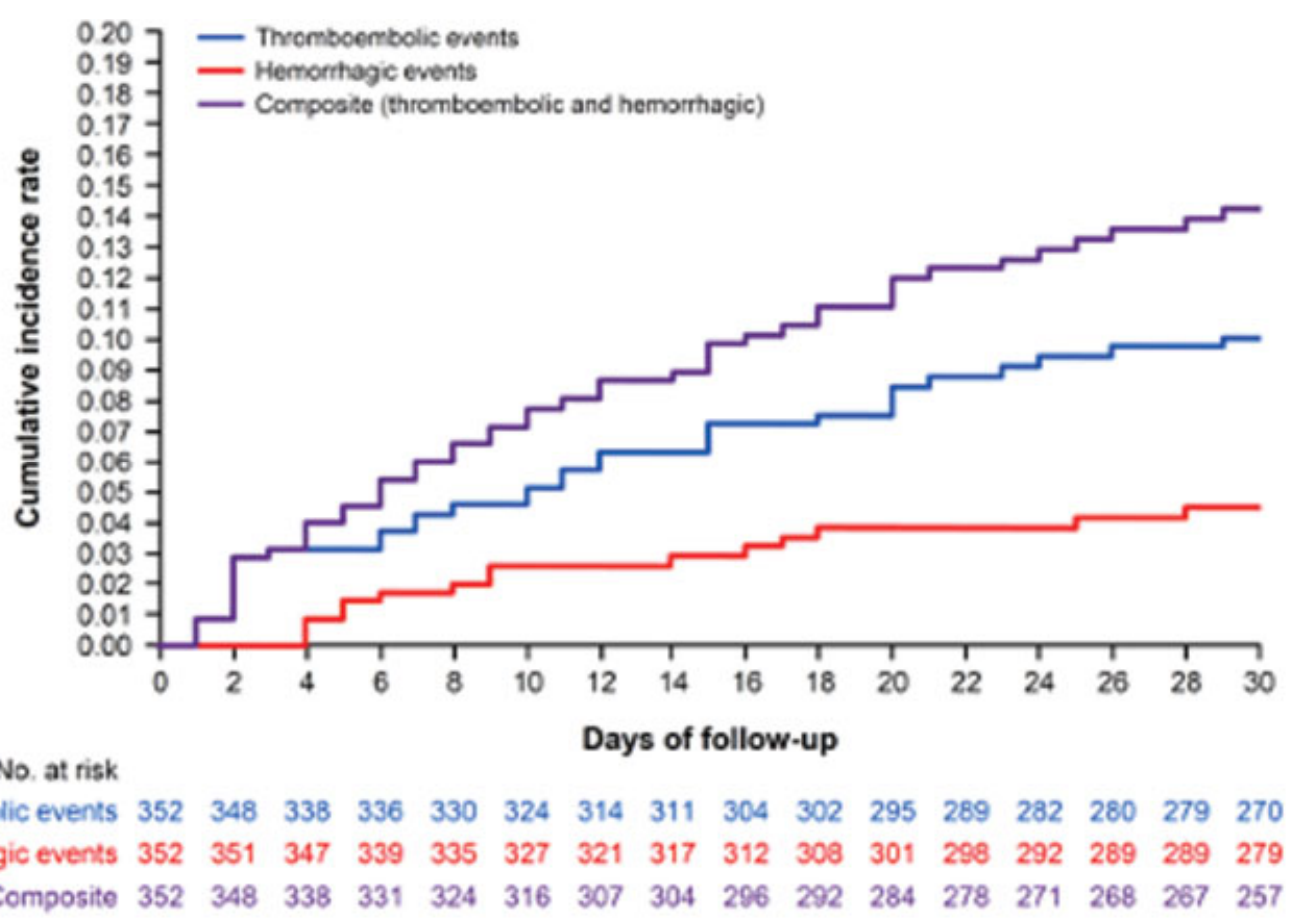

Censored for death and follow-up to Day 30 . The rebleeding events within the first 3 days were excluded from the models.

Fig. 1 Total cumulative incidence rates of thrombotic and rebleeding events (both restarted and not).

(13 cases, 3.7\%) and deep vein thrombosis (13 cases, 3.7\%) were the most frequent thrombotic event types, while myocardial infarctions ( 7 cases, $2.0 \%$ ), pulmonary embolism ( 5 cases, $1.4 \%$ ), and transient ischemic attacks ( 1 case, $0.3 \%$ ) were less common (-Supplementary Table $\mathbf{S 1}$ [available in the online version]). There were 40 total thrombotic events in 34 patients. The thrombotic event rates in patients with ICH, GI bleeding, and other bleeding were 9.3\% (21 of 227), $6.7 \%$ ( 6 of 90 ), and $20 \%$ ( 7 of 35 ), respectively. No thrombotic events occurred after restart of therapeutic anticoagulation; however, eight thrombotic events ( $8 \%$ of those eventually restarted; $2.3 \%, 8$ of 352 of the entire sample) occurred in patients prior to restart (-Supplementary Table $\mathbf{S 1}$ [available in the online version]).

Bleeding events: The three reviewers (T.J.M., J.W.E., and M. C.) deemed that $29(8.2 \%)$ patients had a serious bleeding event (median time to first event: 3 days; IQR: 1-8 days; -Supplementary Table S1 [available in the online version]) (there were no disagreements in the adjudications). Of the 14 bleeds in the first 3 days, 12 were either extensions of the index bleed or new areas of bleeding discovered on computed tomography within the first 48 hours. Of the other two, one developed melena on day 3 , and the other had post-GI bleeding anemia discovered on day 2. (None of these 14 patients had been restarted on oral anticoagulation at the time of bleeding. Bleeding events occurring within 3 days were not included in the analysis, but the patients who had those bleeding events within the first 3 days were included in the subsequent analysis, i.e., they could still go on to be restarted or not and have bleeding and thrombotic events or death as part of both models.) The remaining 15 bleeding events (4.3\% of 347 still living after day 3 ) were incorporated in the models.
-Fig. 1 illustrates the cumulative thrombotic and bleeding events and a composite of the two in the full cohort.

Death: In the 30-day follow-up period, 49 patients (14\%) died. The median time to death was 12 days (IQR: 8-18; -Supplementary Table S1 [available in the online version]).

\section{Landmark Analysis}

The 14-day landmark analysis included 67 restarted and 234 non-restarted patients (-Table 2 ). In the restarted group, none had thrombotic events, three patients had a bleeding event and three patients died. In the non-restarted group, 12 patients had thrombotic events, two patients had a bleeding event, and 17 patients died. In the unadjusted analysis, restart before the 14-day landmark was not significantly associated with reduced thrombotic events $(p=0.06)$ (-Fig. 2 and -Supplementary Table S5 [available in the online version]). After adjustment for age and bleeding site, analyses at the 14-day landmark found that restart was associated with reduced thrombotic events (HR: 0.112; 95\% CI: 0.001-0.944; $p=0.043$ ) and increased bleeding (HR: 8.39; 95\% CI: 1.13-62.29; $p=0.037$; - Table 2). The composite outcomes and death alone were not significant at 14 days, and in the other analyses done at the day 3 and day 5 landmarks; there were no significant associations between restarting and the study outcomes, including death alone.

\section{Cox Models Using Time-Dependent Exposure to Restart}

In the Cox model, restarting was associated with reduced thrombotic events $(\mathrm{HR}=0.071,95 \% \mathrm{CI}$ : 0.001-0.527; $p=0.004)$, and also with a reduction in the triple composite including death $(\mathrm{HR}=0.384 ; 95 \% \mathrm{CI}: 0.161-0.915 ; p=0.031)$ 
Table 2 Landmark analysis on day 14; multivariate analysis of anticoagulation restart versus non-restart ${ }^{\mathrm{a}}$ (significant findings in red)

\begin{tabular}{|c|c|c|c|c|}
\hline Variable & Coefficient & Standard error & p-Value & $\mathrm{HR}(95 \% \mathrm{Cl})^{\mathrm{b}}$ \\
\hline \multicolumn{5}{|c|}{ Rebleeding: for restart ( $n=67$; \# of events: 3 [4.5\%]); for non-restart $(n=234$; \# of events: 2 [0.9\%]) } \\
\hline Indicator of full OAC restart within 14 days (yes $=1$, no $=0$ ) & 2.128 & 1.023 & 0.037 & $8.394(1.131-62.288)$ \\
\hline Age at screen & 0.070 & 0.062 & 0.263 & $1.072(0.949-1.211)$ \\
\hline Initial primary bleeding site: $\mathrm{ICrH}$ & 0.424 & 1.009 & 0.674 & $1.528(0.211-11.049)$ \\
\hline \multicolumn{5}{|c|}{ Thrombotic event ${ }^{\mathrm{a}}$ : for restart $(n=67 ; \#$ of events: 0 [0.0\%]); for non-restart $(n=234 ; \#$ of events: $12[5.1 \%])$} \\
\hline Indicator of full OAC restart within 14 days (yes $=1$, no $=0$ ) & -2.186 & 1.531 & 0.043 & $0.112(0.001-0.944)$ \\
\hline Age at screen & 0.019 & 0.030 & 0.526 & $1.019(0.966-1.088)$ \\
\hline Initial primary bleeding site: $\mathrm{ICrH}$ & -0.542 & 0.622 & 0.378 & $0.582(0.188-2.073)$ \\
\hline \multicolumn{5}{|c|}{ Death: for restart ( $n=67$; \# of events: 3 [4.5\%]); for non-restart $(n=234$; \# of events: 17 [7.3\%]) } \\
\hline Indicator of full OAC restart within 14 days (yes $=1$, no $=0$ ) & -0.230 & 0.683 & 0.737 & $0.795(0.209-3.029)$ \\
\hline Age at screen & 0.104 & 0.032 & 0.001 & $1.110(1.041-1.183)$ \\
\hline Initial primary bleeding site: ICrH & -0.152 & 0.530 & 0.775 & $0.859(0.304-2.430)$ \\
\hline \multicolumn{5}{|c|}{ Composite of rebleeding and thrombotic event: for restart $(N=67$; \# of events: $3[4.5 \%])$; for non-restart $(N=234 ; \#$ of events: 14 [6.0\%]) } \\
\hline Indicator of full OAC restart within 14 days (yes $=1$, no $=0$ ) & -0.262 & 0.687 & 0.703 & $0.770(0.200-2.958)$ \\
\hline Age at screen & 0.033 & 0.028 & 0.226 & $1.034(0.979-1.092)$ \\
\hline Initial primary bleeding site: $\mathrm{ICrH}$ & -0.227 & 0.554 & 0.682 & $0.797(0.269-2.360)$ \\
\hline \multicolumn{5}{|c|}{$\begin{array}{l}\text { Composite of rebleeding, thrombotic event, and death: for restart ( } n=67 \text {; \# of events: } 6 \text { [9.0\%]); for non-restart }(n=234 \text {; \# of events: } 29 \\
[12.4 \%])\end{array}$} \\
\hline Indicator of full OAC restart within 14 days (yes $=1$, no $=0$ ) & -0.143 & 0.489 & 0.770 & $0.867(0.332-2.261)$ \\
\hline Age at screen & 0.060 & 0.022 & 0.006 & $1.062(1.018-1.108)$ \\
\hline Initial primary bleeding site: $\mathrm{ICrH}$ & -0.108 & 0.397 & 0.786 & $0.898(0.412-1.956)$ \\
\hline
\end{tabular}

Abbreviations: $\mathrm{Cl}$, confidence interval; $\mathrm{HR}$, hazard ratio; ICrH, intracranial hemorrhage; OAC, oral anticoagulation.

${ }^{a}$ Model was fitted by Proc PHREG (SAS9.4) using the proportional hazard Cox model except for outcome thrombotic event.

${ }^{b}$ Firth correction using coxphf() in coxphf package of $\mathrm{R}$ was used for outcome thrombotic event to correct bias due to quasi-complete separation feature in the data.

(-Table 3). There was no association of restart with bleeding or death in this model.

\section{Subgroups}

Neither the landmarks nor Cox time-dependent models had any significant findings in the ICH subgroup.

\section{Discussion}

Our data suggest that restarting may be associated with fewer thrombotic events and increased bleeding. The timedependent Cox model triple composite suggests that the overall trade-off with restart when including death may be beneficial, but this needs to be tested in prospective randomized trials.

There is significant uncertainty about whether and when to restart anticoagulants after major hemorrhage. Thrombotic risk is high in the setting of the bleed itself, particularly in $\mathrm{ICH} .{ }^{18}$ Added to this are patients' high baseline risks (i.e., the reason they were on an anticoagulant before the index bleeding) and the risk of immobility after the bleeding episode. This risk is cumulative, rising over time. ${ }^{19}$ Bleeding risk, particularly hematoma expansion in $\mathrm{ICH}$, is high in the first 72 hours and less common thereafter. ${ }^{1}$ Deciding whether and when to restart anticoagulation must balance those opposing risks in any patient.

Some evidence suggests that restarting anticoagulation after major hemorrhage improves outcomes, reducing thrombotic events and mortality, though with an increase in recurrent bleeding. ${ }^{3-8}$ Observational data to assess therapeutic effects are subject to confounding by indication (e.g., lower risk patients are restarted earlier and more often, leading to better outcomes), as we are in these models. However, such data are important in hypothesis generation. Currently, there are no published randomized clinical trials, though several addressing the question of whether to restart, when to restart or both, with bleeding, thrombosis, and/or composite outcomes, are ongoing or about to begin (SoSTART [NCT03153150], ENRICH-AF [NCT03950076], RESTART tICrH [NCT04229758], and ASPIRE [NCT03907046]). We use these composite outcomes in our analysis as they closely approximate the clinical decision that must be made for restarting anticoagulation, a balance between bleeding and thrombosis, so they are clinically meaningful. Composite outcomes 


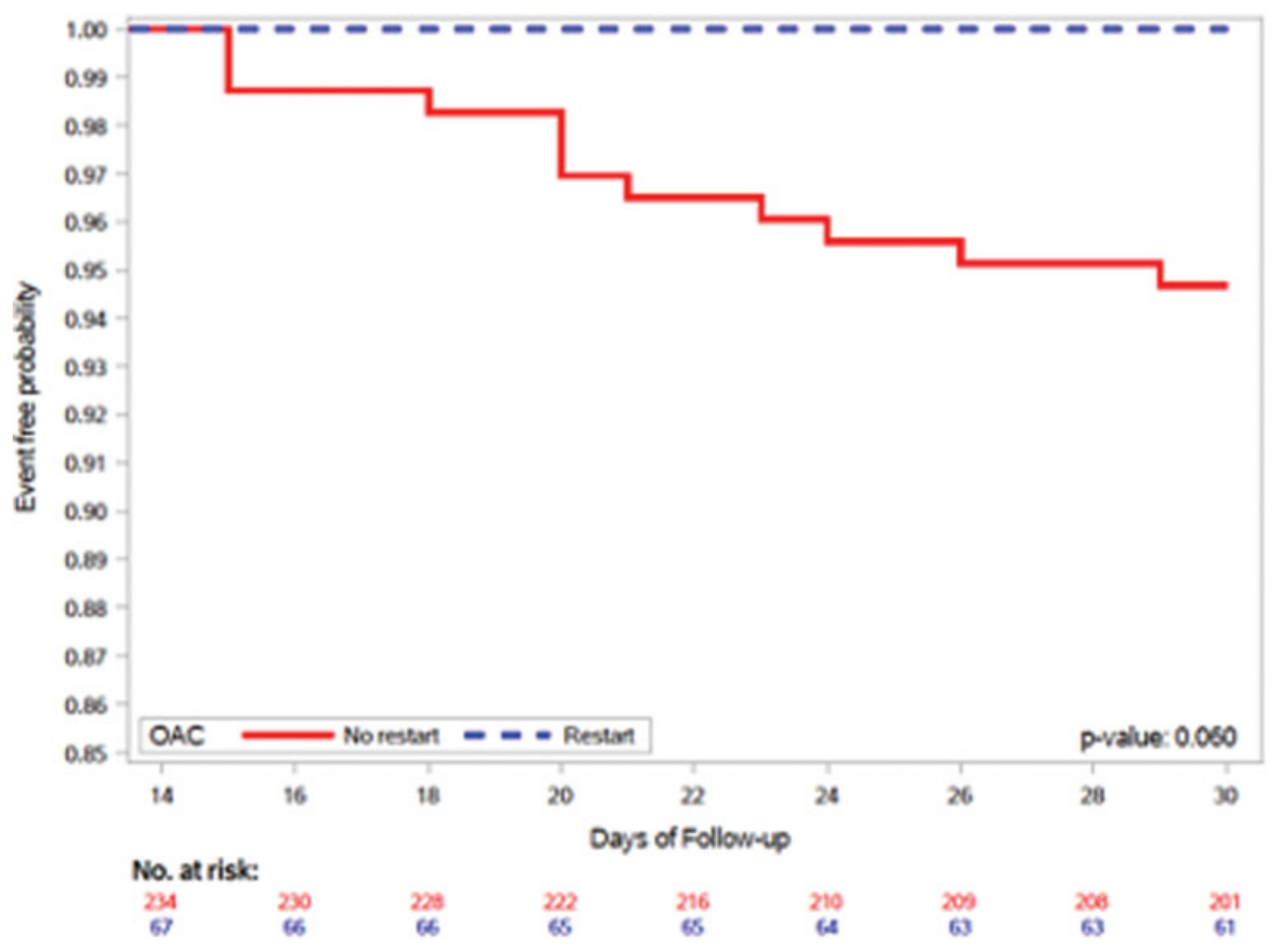

Fig. 2 Kaplan-Meier curve of thrombotic events for landmark analysis on day 14.

also increase power as more events allow for the possibility of larger, more readily detectable differences between groups.

All the adjudicated events in this dataset were serious but did vary in degree of severity. This does cut both ways with bleeding and thrombosis events, i.e., an ICH expansion may be worse than a deep vein thrombosis, but an ischemic stroke is generally worse than a GI bleed. Balancing the severity of bleeding and thrombotic components of composite outcomes has never been done even in prospective trials (though it is a secondary analysis in Restart $\mathrm{TICrH}^{20}$ ) and would not be possible in this model. It requires correlating the events with functional scales such as modified Rankin Scales performed later and does raise the problem of what scales to use across the several outcome events, stroke, myocardial infarction, pulmonary embolism, deep vein thrombosis, GI hemorrhage, recurrent ICH, and other hemorrhage. No single functional scale is validated for all of them, and using specific scales for each makes cross-comparison infeasible. One could simply power on functional scales instead of events, but this requires samples in the thousands, which are simply not feasible in a relatively infrequent disease. (For example, ANNEXA-4 required a global network of 63 sites more than 3 years to accrue 352 anticoagulated major bleeding patients. ${ }^{9}$ ) A sample powered on mortality would have to be quite a bit larger still.
A strength of this analysis is that anticoagulant reversal trials offer a degree of rigor for secondary events such as thrombotic complications, as these are carefully captured primary safety events. Unlike other retrospective analyses using medical records or databases, the present analysis uses patient-level data gathered prospectively with independent academic adjudication of thrombotic events and an equal follow-up period. ${ }^{9}$ Retrospective hospital medical record analyses are more difficult as patients are hospitalized for varying periods creating uneven surveillance (i.e., whether a patient had an event after discharge may not be known).

Selection bias would have influenced our results-since clinicians made a decision to restart based on the patient's individual characteristics. Clinicians were less likely to restart anticoagulation in older patients and those with central nervous system bleeding. Although we adjusted for these variables in our analysis, we cannot rule out residual confounding. For example, we have no insight into the factors clinicians considered in the restart decision, such as thrombotic risk or patient preference, and we have no data on medication compliance in those who were restarted. One can infer some restart clinical reasoning from the structure of the models. In the landmark models, patients with events prior to the landmark time point were excluded, so these restart decisions were for prevention of thrombosis. The Cox model compares events per time restarted and not restarted, so it 
Table 3 Multivariate analysis of time-dependent Cox proportional hazards model of restart ${ }^{\mathrm{a}}$ (significant findings in red)

\begin{tabular}{|c|c|c|c|c|}
\hline Variable & Coefficient & Standard error & $p$-Value & HR $(95 \% \mathrm{Cl})^{\mathrm{b}}$ \\
\hline \multicolumn{5}{|c|}{ Multivariate analysis of outcome rebleeding } \\
\hline Indicator of restart OAC & 0.512 & 0.721 & 0.478 & $1.668(0.406-6.857)$ \\
\hline Age at screen & -0.002 & 0.025 & 0.931 & $0.998(0.951-1.047)$ \\
\hline Initial primary bleeding site: $\mathrm{ICrH}$ & 0.242 & 0.596 & 0.685 & $1.274(0.396-4.093)$ \\
\hline \multicolumn{5}{|c|}{ Multivariate analysis of outcome thrombotic event ${ }^{\mathrm{b}}$} \\
\hline Indicator of restart OAC & -2.651 & 1.475 & 0.004 & $0.071(0.001-0.527)$ \\
\hline Age at screen & 0.011 & 0.017 & 0.500 & $1.011(0.980-1.048)$ \\
\hline Initial primary bleeding site: $\mathrm{ICrH}$ & -0.449 & 0.361 & 0.216 & $0.638(0.322-1.314)$ \\
\hline \multicolumn{5}{|l|}{ Multivariate analysis of outcome death } \\
\hline Indicator of restart OAC & -1.040 & 0.619 & 0.093 & $0.354(0.105-1.191)$ \\
\hline Age at screen & 0.066 & 0.018 & $<0.001$ & $1.068(1.031-1.107)$ \\
\hline Initial primary bleeding site: $\mathrm{ICrH}$ & -0.162 & 0.320 & 0.613 & $0.850(0.454-1.594)$ \\
\hline \multicolumn{5}{|c|}{ Multivariate analysis of composite outcome of rebleeding and thrombotic events } \\
\hline Indicator of restart OAC & -1.032 & 0.623 & 0.098 & $0.356(0.105-1.208)$ \\
\hline Age at screen & 0.011 & 0.015 & 0.445 & $1.011(0.983-1.040)$ \\
\hline Initial primary bleeding site: $\mathrm{ICrH}$ & -0.300 & 0.313 & 0.337 & $0.740(0.401-1.367)$ \\
\hline \multicolumn{5}{|c|}{ Multivariate analysis of composite outcome of rebleeding, thrombotic events, and death } \\
\hline Indicator of restart OAC & -0.958 & 0.443 & 0.031 & $0.384(0.161-0.915)$ \\
\hline Age at screen & 0.031 & 0.012 & 0.011 & $1.031(1.007-1.056)$ \\
\hline Initial primary bleeding site: $\mathrm{ICrH}$ & -0.227 & 0.239 & 0.343 & $0.797(0.499-1.274)$ \\
\hline
\end{tabular}

Abbreviations: $\mathrm{Cl}$, confidence interval; $\mathrm{HR}$, hazard ratio; $\mathrm{ICrH}$, intracranial hemorrhage; OAC, oral anticoagulation.

${ }^{a}$ Model was fitted by Proc PHREG (SAS9.4) using the proportional hazard Cox model except for outcome thrombotic event.

${ }^{b}$ Firth correction using coxphf() in coxphf package of $\mathrm{R}$ was used for outcome thrombotic event to correct bias due to quasi-complete separation feature in the data.

would include restarting both for prevention and treatment of thrombotic disease (treatment meaning the patient has already had a thrombotic event post major bleed forcing the restart decision). However, our data are merely hypothesisgenerating and cannot be considered reliable enough to guide clinical practice.

We excluded bleeding events in the first 3 days. This exclusion is supported by the natural history of anticoagulant-associated bleeding, particularly in the central nervous system. Hawryluk et al ${ }^{1}$ extracted data from 63 case reports and series on 492 anticoagulated central nervous system hemorrhage patients, finding a rebleeding rate of $8 \%$ and a thrombotic event rate of $6 \%$. Temporal mapping showed most rebleeding events occurred in the first 72 hours, while most thrombotic events occurred from 3 days to 1 week after the patient's presentation with bleeding. Including these early bleeding events, nearly all of which were extensions of the index bleeding, clouds the model with regard to restarting.

Restart timing is an important and as yet unanswered question. Current retrospective evidence, expert opinion, and clinical practice on timing are highly variable. Recommendations range from 3 days $^{1}$ to 30 weeks, ${ }^{2}$ though the American Stroke Association guideline states $\geq 1$ week with multiple caveats. ${ }^{21}$ Majeed et al $^{2}$ retrospectively evaluated
234 ICH patients, 59 of whom restarted warfarin. Cox models found a restart interval of 10 to 30 weeks minimized a composite outcome. Our time-dependent Cox model attempts to simulate timing's influence by comparing time restarted versus not in terms of the outcomes and produces an adjusted HR (-Table 3 ). The landmark approach is an attempt to incorporate timing by setting points by which a certain number of (event-free) patients have been restarted and comparing outcomes thereafter. This can be represented as a Kaplan-Meier curve (-Fig. 2). It is not the same thing as finding that a time point is a "best" restart time. This can only be accomplished in randomized models comparing different timing strategies (such as the Restart TICrH trial ${ }^{20}$ ) in which each group has a specified restart day. Patients in our dataset could be restarted at any time in the 30-day follow-up. The more specific one gets to a certain time interval in the dataset, the less restarted patients there are and thus the less power to detect differences in outcomes. This probably explains why the 14-day landmark had the peak power to detect differences. It was enough time for enough patients to be restarted to have a larger group for comparison with sufficient time remaining for outcome differences in groups to accumulate and potentially differ. Later landmark time points reduce power in a slightly different way. The data terminate at 30 days, so a restart landmark at 3 weeks, for 
example, allows only 1 week for outcomes to accumulate and potentially differ. Less outcomes lead to smaller differences between groups and thus less power to detect those differences.

It is important to categorize these findings in terms of the types of bleeding, i.e., spontaneous intracerebral hemorrhage versus traumatic ICH versus GI and other major hemorrhage. Each has very different pathophysiologies and risks and consequences of rebleeding with restart of anticoagulation, i.e., rebleeding in the cranium is life-threatening while rebleeding in the gut may not be. Whether and when to restart must account for these variables, and this analysis cannot speak to them as we include patients from all three categories.

Our findings modestly support an emerging body of literature that restarting may confer a benefit to patients. Park et $a^{22}$ retrospectively analyzed 428 atrial fibrillation patients with a history of $\mathrm{ICH}$, finding benefit in a composite outcome for restarting at $\geq 2$ weeks. It is important to note that Park et al followed patients for $>3$ years on average while our study was limited to 30 days, during which it is more difficult to detect differences in relatively infrequent events.

This analysis has important limitations. Because all patients received andexanet, we cannot speak to any thrombotic risk associated with this agent, so we cannot be sure these findings would translate to patients who did not receive andexanet. Our analysis is underpowered to detect the likely differences in thrombotic events and bleeding at restart intervals across the 30-day follow-up period. Since this secondary analysis is limited to data from ANNEXA-4, there could be uncollected data points that influence bleeding, thrombosis, death, and/or restart behavior. Posthoc analyses have known sources of bias (e.g., survivorship and selection, and unknown/uncollected confounders) that temper our findings. Bleeding events were drawn from investigator-reported SAE files, which may not include all bleeding complications, though should include all serious ones. We only found significant associations when we treated all index major bleedings as a single group, e.g., the ICH subgroup analyses had no significant findings. Severity of ICH and its surrogates such as hematoma volume likely influence restart decisions, but we did not detect it in these models. Also, spontaneous ICH, traumatic ICH, GI, and other bleeding types all have differing risks. This likely influences the odds and timing of restarting decisions and secondary outcomes.

\section{Conclusion}

Restarting therapeutic oral anticoagulation after major hemorrhage was associated with decreased risk of thrombotic events and increased risk of bleeding in this highly selected ANNEXA-4 clinical trial population. There is modest evidence for a net benefit when combining thrombosis, hemorrhage, and death into a composite. These results should be viewed cautiously as hypothesis-generating, as they are retrospective, and the reasons for restarting patients could introduce bias. They may support the conduct of randomized clinical trials to test if restarting anticoagulation after acute major bleeding has a net clinical benefit.

\section{What is known about this topic?}

- The relationship between restarting anticoagulation after major hemorrhage and clinical outcomes is unclear.

\section{What does this paper add?}

- In a posthoc analysis of a prospective, 352-patient anticoagulant reversal trial, no thrombotic events occurred after restarting anticoagulation, but bleeding increased, and restarting was associated with a decrease in a composite of thrombotic events, rebleeding, and death, suggesting a net benefit.

\section{Author Contributions}

T.J.M. had full access to all the data in the study and takes responsibility for the integrity of the data and the accuracy of the data analysis. Concept and design: T.J.M., B.K., L.X., and S.J.C. Acquisition, analysis, and interpretation of data: All authors. Drafting of the manuscript: T.J.M. Critical revision of the manuscript for important intellectual content: All authors. Statistical analyses: B.K. and L.X.

\section{Data Sharing Statement}

Alexion will consider requests for disclosure of clinical study participant-level data provided that participant privacy is assured through methods like data de-identification, pseudonymization, or anonymization (as required by applicable law), and if such disclosure was included in the relevant study informed consent form or similar documentation. Qualified academic investigators may request participant-level clinical data and supporting documents (statistical analysis plan and protocol) pertaining to Alexion-sponsored studies. Further details regarding data availability and instructions for requesting information are available in the Alexion Clinical Trials Disclosure and Transparency Policy at https://alexion. com/our-research/research-and-development.

Link to Data Request Form (https://alexion.com/contactalexion/medical-information).

\section{Funding}

This study was funded by Portola Pharmaceuticals, Inc., South San Francisco, California, United States, now Alexion Pharmaceuticals, Inc., Boston, Massachusetts, United States, following acquisition by Alexion. Editorial support was provided by Cello Health Communications and funded by Alexion Pharmaceuticals, Inc.

\section{Conflict of Interest}

T.J.M.: CSL Behring: consulting; Portola: grant support, steering committee; Octapharma: steering committee. B.K.: none. P.Y.: Portola: employee. S.M.: Daiichi Sankyo, 
Bayer, Aspen Pharma: grant support; Bristol Myers Squibb/Pfizer, Boehringer Ingelheim, Portola: advisory board; Portola: lecture fees; AbbVie: adjudication committee. J.B.-W.: Bayer, Daiichi Sankyo: grant support, lecture fees, advisory board fees; Pfizer: grant support; Portola: lecture fees, advisory board fees. J.W.E.: AstraZeneca, Bayer, Boehringer Ingelheim, Bristol Myers Squibb/Pfizer, Daiichi Sankyo, Eli Lilly, GlaxoSmithKline, Janssen, Sanofi-Aventis: grant support, honoraria. M.C.: Bristol Myers Squibb Canada, Asahi Kasei, Servier Canada, Precision Biologics, Hemostasis Reference Laboratory, CSL Behring: advisory board; Alexion, Pfizer, CSL Behring, Diagnostica Stago: educational funding; Bayer, Leo Pharma: grant support; Daiichi Sankyo, Bayer, Octapharma: data safety monitoring board. L.X.: None. P.V.: Bayer Healthcare, Boehringer Ingelheim, Daiichi Sankyo, Pfizer, Bristol Myers Squibb, Leo Pharma: grant support, consulting, lecture fees; Boehringer Ingelheim: adjudication committee; Anthos, Janssen, Portola: consulting. D.M.S.: Bayer, Bristol Myers Squibb/Pfizer, Servier Canada, Novartis, Leo Pharma, Aspen Pharma, Portola: consulting. S.J.C.: Portola, Bristol Myers Squibb, Bayer, Daiichi Sankyo: grant support, consulting; Javelin: consulting.

\section{References}

1 Hawryluk GW, Austin JW, Furlan JC, Lee JB, O’Kelly C, Fehlings MG. Management of anticoagulation following central nervous system hemorrhage in patients with high thromboembolic risk. J Thromb Haemost 2010;8(07):1500-1508

2 Majeed A, Kim YK, Roberts RS, Holmström M, Schulman S. Optimal timing of resumption of warfarin after intracranial hemorrhage. Stroke 2010;41(12):2860-2866

3 Pennlert J, Overholser R, Asplund K, et al. Optimal timing of anticoagulant treatment after intracerebral hemorrhage in patients with atrial fibrillation. Stroke 2017;48(02):314-320

4 Zhou Z, Yu J, Carcel C, et al. Resuming anticoagulants after anticoagulation-associated intracranial haemorrhage: systematic review and meta-analysis. BMJ Open 2018;8(05):e019672

5 Nielsen PB, Larsen TB, Skjøth F, Lip GY. Outcomes associated with resuming warfarin treatment after hemorrhagic stroke or traumatic tracranial hemorrhage in patients with atrial fibrillation. JAMA Intern Med 2017;177(04):563-570

6 Nielsen PB, Larsen TB, Skjøth F, Gorst-Rasmussen A, Rasmussen LH, Lip GY. Restarting anticoagulant treatment after intracranial hemorrhage in patients with atrial fibrillation and the impact on recurrent stroke, sortality, and bleeding: a nationwide cohort study. Circulation 2015;132(06):517-525
7 Murthy SB, Gupta A, Merkler AE, et al. Restarting anticoagulant therapy after intracranial hemorrhage: a systematic review and meta-analysis. Stroke 2017;48(06):1594-1600

8 Little D, Chai-Adisaksopha C, Hillis C, et al. Resumption of anticoagulant therapy after anticoagulant-related gastrointestinal bleeding: a systematic review and meta-analysis. Thromb Res 2019;175:102-109

9 Connolly SJ, Crowther M, Eikelboom JW, et al; ANNEXA-4 Investigators. Full study report of andexanet alfa for bleeding associated with factor Xa inhibitors. N Engl J Med 2019;380(14):1326-1335

10 Lin DY, Dai L, Cheng G, Sailer MO. On confidence intervals for the hazard ratio in randomized clinical trials. Biometrics 2016;72 (04):1098-1102

11 Peto R, Pike MC, Armitage P, et al. Design and analysis of randomized clinical trials requiring prolonged observation of each patient. II. analysis and examples. Br J Cancer 1977;35(01):1-39

12 Heinze G, Schemper M. A solution to the problem of monotone likelihood in Cox regression. Biometrics 2001;57(01):114-119

13 Firth D. Bias reduction of maximum likelihood estimates. Biometrika 1993;80(01):27-38

14 R Core Team. 2017R: A language and environment for statistical computing. $\mathrm{R}$ Foundation for Statistical ComputingVienna, Austria. Available at: https://www.R-project.org/

15 Suissa S. Immortal time bias in pharmaco-epidemiology. Am J Epidemiol 2008;167(04):492-499

16 Mi X, Hammill BG, Curtis LH, Lai EC, Setoguchi S. Use of the landmark method to address immortal person-time bias in comparative effectiveness research: a simulation study. Stat Med 2016;35(26):4824-4836

17 Dafni U. Landmark analysis at the 25-year landmark point. Circ Cardiovasc Qual Outcomes 2011;4(03):363-371

18 Goldstein JN, Fazen LE, Wendell L, et al. Risk of thromboembolism following acute intracerebral hemorrhage. Neurocrit Care 2009; 10(01):28-34

19 Milling TJ Jr, Refaai MA, Goldstein JN, et al. Thromboembolic events after vitamin $\mathrm{k}$ antagonist reversal with 4-factor prothrombin complex concentrate: exploratory analyses of two randomized, plasmacontrolled studies. Ann Emerg Med 2016;67(01):96-105.e5

20 Milling TJ, Warach S, Johnston SC, et al. Restart TICrH Trial Design: an adaptive randomized trial of time intervals to restart direct oral anticoagulants after traumatic intracranial hemorrhage. J Neurotrauma 2021. Doi: 10.1089/neu.2020.7535

21 Kernan WN, Ovbiagele B, Black HR, et al; American Heart Association Stroke Council, Council on Cardiovascular and Stroke Nursing, Council on Clinical Cardiology, and Council on Peripheral Vascular Disease. Guidelines for the prevention of stroke in patients with stroke and transient ischemic attack: a guideline for healthcare professionals from the American Heart Association/American Stroke Association. Stroke 2014;45(07):2160-2236

22 Park YA, Uhm JS, Pak HN, Lee MH, Joung B. Anticoagulation therapy in atrial fibrillation after intracranial hemorrhage. Heart Rhythm 2016;13(09):1794-1802 